\title{
Ambient Energy Sources, Applications and Ambient Energy Harvesting in Cognitive Radio
}

\author{
Aparna Singh ${ }^{1}$, Anu Shrivastava ${ }^{2}$ \\ Assistant Professor, Department of Electronics and Communication Engineering, University Institute of Technology, RGPV \\ M. Tech Student, Department of Electronics and Communication Engineering, University Institute of Technology, RGPV
}

\begin{abstract}
Reinforced concrete (RC) structure using externally bonded fiber reinforced polymer (FRP) components has become a very worldwide practice, extensively accepted by recent design codes. In particular, the flexural strength of a reinforced concrete beam can be broadly increased by application of carbon, glass FRP sheets adhesively bonded to the tension face of the beam. In this experimental study, a total of nine beam specimens $(1000 \mathrm{~mm} \times 150 \mathrm{~mm} \times 200 \mathrm{~mm})$ of $\mathrm{M} 20$ grade concrete are casted and preloaded with $75 \%$ of ultimate load by two point load method, later retrofitted with hybrid laminates $(G F R P+C F R P)$ of different thicknesses at $U$-wrap bonding technique used to examine the flexural behavior of $R C$ beam. . A comparative study is to be made with the test results.
\end{abstract}

Keywords: Fiber Reinforced Polymer (FRP), carbon, glass, energy absorption capacity, ductility, debonding

\section{Introduction}

The future of digital communication is predicted as one of hyper connectivity powered by the Internet of things where every device is connected to other devises via a wireless network, where every object we use would be designed to become an information source, information that would be generated locally and used among other things to simplify our lives.Future from the perspective of energy in such devices would be to design those devises to also generate energy locally. What if we could utilize every possible energy source available to harvest energy? From mechanical movements, thermal variations to using electromagnetic energy abundantly available in urban environments. Cognitive radio, defined as a radio that can adapt to its environment, based systems are the best answer to implement such networks. This literature review lists possible green energy sources for the purposes of ambient energy harvesting, their existing applications and the analyses the possibility of their implementation in cognitive radios.

Harvesting energy from non dedicated sources in the environment is known as ambient energy harvesting or energy scavenging. Broadly the sources are Ambient Heat, Acoustic Noise, Temperature variations, RF energy, Airflow, Vibration, Piezoelectric energy: human activity. Additionally, chemical and biological sources and radiation can be considered as ambient energy sources.In this paper majorambient energy sources are described in brief and the different ways they have been harvested are mentioned as their applications. In the end RF energy harvesting is described in detail in the view of its implementation in cognitive radio systems.

Ambient Heat: The so called ambient heat is the solar energy temporarily stored in the mass and atmosphere of the earth as well as the flora and fauna. Ambient heat is peculiar in that it can be harnessed only indirectly by means of suitable energy exchangers (heat pumps) [2]. A heat pump is a device that provides heat energy from a source of heat to a destination called a "heat sink". Heat pumps are designed to move thermal energy opposite to the direction of spontaneous heat flow by absorbing heat from a cold space and releasing it to a warmer one. Among all the forms of renewable energy sources ambient heat is more than any dependent on auxiliary energy (e.g. electrical energy or fuels) for its utilization according to the thermal dynamic principle of the heat pump [2]. These are used as an alternative of air conditioners and can be used on a standalone basis in home applications in moderate climates. These can be used as a supplement in industrial environments and data centers for cooling.

Airflow: Traditionally wind energy has been utilized in wind turbines where the wind turns the blades of a fan which turn a shaft which goes into a gearbox which increases the rotation speed. A generator converts the rotational energy into electrical energy. A transformer converts the electricity from around 700 Volts (V) to the right voltage for distribution, typically $33,000 \mathrm{~V}$.

A new concept known as Windstalk has been proposed by New York design firm Atelier DNA. This uses 1,203 carbon fiber reinforced resin poles, which stand 55 meters (180 feet) high and are anchored to the ground in concrete bases that range between 10 and 20 meters (33-66 ft) in diameter. The poles, which measure $30 \mathrm{~cm}$ (12 in.) in diameter at the base, tapering up to a diameter of $5 \mathrm{~cm}$ ( 2 in.) at the top, are packed with a stack of piezoelectric ceramic discs.[1] Between the discs are electrodes that are connected by cables that run the length of each pole - one cable connects the even electrodes, while another connects the odd ones.

So, instead of relying on the wind to turn a turbine to generate electricity, when the pole sways in the wind, the stack of piezoelectric discs are compressed, generating a current through the electrodes.

As a way to maximize the amount of electricity the Windstalk farm would generate, the concept also places a torque generator within the concrete base of each pole. As the poles sway, fluid is forced through the cylinders of an array of current generating shock absorbers to convert the 


\section{International Journal of Science and Research (IJSR) \\ ISSN (Online): 2319-7064 \\ Index Copernicus Value (2013): 6.14 | Impact Factor (2015): 6.391}

kinetic energy of the swaying poles into electrical energy. This has not been implemented yet.

Acoustic Noise: Acoustic energy harvesting is very challenging because of poor coupling efficiency of acoustic waves. A plane wave in air at the threshold of human pain

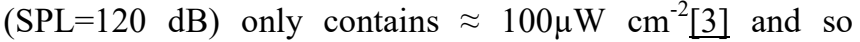
research efforts in this category have been rare. Mainly, two types of acoustic energy harvestershave been developed and reported in literature: electromagnetic and piezoelectric based. The power produced by the reported piezoelectric acoustic energy harvesters ranges from $0.68 \mathrm{pW}$ to $30 \mathrm{~mW}$; however, the power generation of the developed electromagnetic acoustic energy harvesters is in the range of $1.5-1.96 \mathrm{~mW}$.[4] The overall size of most of the developed piezoelectric and electromagnetic AEHs are quite comparable and in millimeter scale. The resonant frequencies of electromagnetic acoustic energy harvesters are on the lower side $(143-470 \mathrm{~Hz})$, than that of piezoelectric acoustic energy harvesters (146 Hz$16.7 \mathrm{kHz}) \cdot[4]$

Acoustic energy harvesters made using electromechanical Helmholtz resonator (EMHR) consists of an orifice, cavity, and a piezoelectric diaphragm. Acoustic energy is converted to mechanical energy when sound incident on the orifice generates an oscillatory pressure in the cavity, which in turns causes the vibration of the diaphragm. The conversion of acoustic energy to electrical energy is achieved via piezoelectric transduction in the diaphragm of the EMHR. The diaphragm is coupled with energy reclamation circuitry to increase the efficiency of the energy conversion.

Another acoustic energy harvester is made using sonic crystal and the piezoelectric material. A point defect is created by removing a rod from a perfect sonic crystal. The point defect in the sonic crystal acts as a resonant cavity, and the acoustic waves at the resonant frequency of the cavity can be localized in the cavity. The power generation from acoustic energy is based on the effect of the wave localization in the cavity of the sonic crystal and the direct piezoelectric effect of the piezoelectric material.

Temperature Variations: Seebeck effect is used to convert a temperature gradient into electrical current. According to it if an electrical path consisting of two different conductors is subjected to a temperature differential, electron flow occurs from higher temperature region to lower temperature region resulting in a DC current. Thermoelectric generators (TEG) are used to harvest energy from this ambient source using Seebeck effect. More accurate for our pruposeswould be micro thermoelectric (TE) energy generators because of abundant but low heat sources present in the environment. Micro TEG es exist in two main configurations: cross plane and in plane depending on the direction of heat flow through the thermocouples and the orientation to the substrate surface. In the cross plane configuration heat flows perpendicular to the substrate and in the in plane configuration heat flow is parallel to the substrate. The latter has the advantage of generating relatively higher voltages for the same temperature differences owing to longer length of the thermocouples. Additionally an array of TEGs can be formed to generate higher output power for the same temperature difference than a single TEG. A single TEG generates power in the microwatts range.

Vibrational Energy: Most of the efforts in vibrational energy harvesting have been focused on single frequency sinusoidal input excitation to drive an energy harvesting device. But in real world applications the input may contain multiple frequencies or might as well be white noise. So single frequency based vibrational energy harvesting devices are very inefficient in real world. One of the energy harvesting device is electrodynamic vibrational energy harvester in which external vibrations are used to create relative motion between a magnet and a conducting coil. The flux changes in the coil induces an emf across the conducting coil. It has an efficiency of $25-50 \%$ and the output power is in the range of 4-800 microWatts.[5]

Piezoelectric Energy: This method alters mechanical energy into electrical energy by straining a piezoelectric material. Strain or deformation of a piezoelectric material causes charge separation across the device, producing an electric field and consequently a voltage drop proportional to the stress applied. The power produced by the reported piezoelectric ambient energy harvesters ranges from 0.68 $\mathrm{pW}$ to $30 \mathrm{~mW}[6]$

Human activity: Energy derived from human activity appears to be the most underutilized ambient energy resource. Humans are capable of operating machines (e.g., cranks, levers, springs, etc.) to generate mechanical power ranging from a few milliwatts to more than 500W[7]. The upper end of this range corresponds to athletic performance and can be sustained for relatively short periods.

Shoe inserts are the most common application in this category. The process of walking provides oscillatory pressure to activate a number of mechanisms built into shoes or boots. Small amounts of power are available by simply inserting available piezoelectric actuators in the soles of footwear. Ideally, the best piezo materials might produce over $1 \mathrm{~J}$ per step.[7] An alternative to piezoelectric based circuit is miniature electromagnetic generator. Since walking produces varying levels of output as an individual goes about and rests between normal duties, mechanical to electrical energy conversion is likely to occur at vastly different rates throughout the day. Therefore, all concepts in this category appear to require battery storage to accumulate this energy.

Another utilization of human movements has been created by London-based Pavegen, they have built energyharvesting tiles based on a hybrid piezoelectric technology that transform footsteps into electricity. Each tile has a capacity of 6 watts, in order to use the tile se full capacity, there needs to be a constant flow of about 50 steps / minute. The Pavegen technology is practical in areas with high foot traffic. Moreover, the tiles are made from recycled rubber and other recyclable materials.

Yet another is the conversion of kinetic energy to electricity using Faradays law. One example of it would be "SOCCKET" it"s a football which works as a portable generator. Soccket was developed by the company 


\section{International Journal of Science and Research (IJSR) \\ ISSN (Online): 2319-7064}

Index Copernicus Value (2013): 6.14 | Impact Factor (2015): 6.391

Uncharted Play. The concept involves a mechanical oscillator arrangement which consists of a coil, and a movable cantilever with magnet on one end and balancing weight on the other end. As the football is played with, the motion of the magnet changes the magnetic flux crossing the coil and an emf is induced which is then stored in a battery. The company says 30 minutes of play time is enough to generate three hours of energy on an LED light supplied by them. Exact power details are not specified though.

RF Energy: Wireless energy transfer and harvesting of RF energy can be classified into two categories: near-field and far-field systems.

\section{NEARFIELD SYSTEMS (distance $<0.1 \lambda$ ) utilize electric/magnetic induction to transfer power wirelessly and usually have a high transfer efficiency (more than 80\%) within a wavelength from the source [8]-[10], For these systems to operate efficiently, the magnetic coupling between the source and device coils must be large for proper operation. From an implementation point of view it means that the primary coil (source) and secondary coil (device) must be located in close proximity and require careful positioned with respect to one another.}

The effect of resonance has been used to harvest energy using induction for relatively longer distances or "mid range" distances[11]. This non-radiative approach uses resonance to enhance the efficiency of the energy transfer. In some instances, this technology is also referred to as "magnetic resonance", or "highly resonant" wireless energy transfer or "highly resonant" wireless power transfer (HRWPT), and it is often contrasted to "induction" for its ability to efficiently transfer power over a range of distances and with position and orientation offsets. High quality factor resonators enable efficient energy transfer at lower coupling rates, i.e., at greater distances and/or with more positional freedom than is otherwise possible [12-15]Resonant wireless power transfer systems typically operate in the low $\mathrm{MHz}$ range (approximately $1-20 \mathrm{MHz}$ ). At frequencies below this range, it is difficult to realize high-Q resonant coils with dimensions appropriate for mobile devices, whereas at higher frequencies, the shorter wavelengths limit the spatial extent of the near-field coupling. Electromagnetic energy decays at a rate of $60 \mathrm{~dB} /$ decade in near-field region [16] thus this approach is suitable for short-range energy harvesting systems.

A power harvesting metamaterial has been created to harvest near field RF energy. Metamaterials are composed of subwavelength particles that exhibit bulk properties that are different from their individual components. The power harvesting metamaterial is an example of a functional metamaterial that may be suitable for a wide variety of applications that require power delivery to any active components integrated into the metamaterial. Its implemented using a resonant induction mechanism in a split ring resonator circuit(SRR).A maximum of $36.8 \%$ of the incident power from a $900 \mathrm{MHz}$ signal was experimentally rectified by an array of metamaterial unit cells [17]. SRR can exhibit a strong magnetic resonance to EM wave with high quality factor (Q) at resonance. This makes it an excellent candidate for near-field resonant power/data transfer [18]In addition to microwaves, researchers are looking into harvesting power froma metamaterial array from a range of frequencies, as well as vibrations and sound waves.

A matter of concern and limitation with these technologies is that strong reactive near-fields of wireless power systems may induce high fields in the body tissue of persons in their closest vicinity.

Farfield Systems: Radiated far-field power can propagate to a longer distance compared to near-field inductively coupled power due to a different attenuation rate. Farfield energy attenuates at $20 \mathrm{~dB} /$ decade, which is a much smaller rate compared to that of near-field energy [19]. Far field energy harvesters are based on radiative RF energy transfer which uses the transmission and reception of radio waves. Wireless energy-harvesting devices for ambient signals far away from the RF sources have been reported in [20], [21], and [22]-[25].

The high density of RF energy in an urban area has been considered an energy source [23]-[25]. The wireless signals, such as TV, cellular, global system for mobile (GSM), and radio signals, are spread over multiple frequencies in urban areas although the power level of each signal could be as low as $40 \mathrm{dBm}$. Multiband antennas, optimized rectennas, and power management modules for each frequency band were designed and integrated to collect efficiently the ambient RF energy. A magnetic material loaded antenna was utilized to harvest energy in these reported efforts. The reported works in [22]-[25] demonstrated the feasibility of powering low-power electronics from prebuilt wireless infrastructure systems, such as a communication system or a broadcasting system, without a battery.

Many reported efforts have used complementary metaloxide-semiconductor (CMOS) technology to harvest power at $U H F$ radio-frequency identification (RFID) band utilizing conventional RFID standards [26]-[29]. The RF energy harvesters based on CMOS technology are much more compact than board level designs, but they are challenging to optimize at various frequency bands and various input power levels once a design is fixed.

A rectifier with a dc-dc converter topology for the cellular frequency band at $1.96 \mathrm{GHz}$ was proposed in [20]. The reported RF-dc conversion efficiency was more than $60 \%$ when the input power level was higher than $15 \mathrm{dBm}(30 \mathrm{~W})$ and the RF source was $50 \mathrm{~m}$ away from the energy harvester, a shorter distance compared to other similar harvesters. A lithium battery was charged by the harvested RF power.

Recently, an ambient digital TV signal at the UHF band has been harvested and autonomous operation of a sensor platform has been demonstrated in [20] and [21]. In this research effort, a high-gain broadband antenna was utilized to harvest a sufficient amount of power to turn on an embedded microcontroller or a sensor and implement a truly autonomous operability. Digital TV signals within 512-566$\mathrm{MHz}$ frequency bands were captured, rectified, and stored in a capacitor to drive the embedded systems from $6.3 \mathrm{~km}$ 


\section{International Journal of Science and Research (IJSR) \\ ISSN (Online): 2319-7064 \\ Index Copernicus Value (2013): 6.14 | Impact Factor (2015): 6.391}

away from the digital TV broadcasting station. A charge pump circuit was optimized to rectify and create $1.8-3.3 \mathrm{~V}$ from ambient RF power (below $25 \mathrm{dBm}$ ) at the UHF band.

In green cognitive radio(CR)systems, RF harvesting is an energy form of particular potential because the green CR can transmit data on the idle spectrum while harvesting energy from the busy spectrum [30] Another advantage of RF energy is the simultaneous transfer of wireless information and power [31]

In $\mathrm{CR}$, RF energy harvesting implementation can be modelled in two ways:

1) Separated energy harvester and information receiver. In this a dedicated, separated energy harvester is present because of which simultaneous energy harvesting and consumption (sensing, transmission) is possible.Two separate receiving antennas are required for data detection and energy harvesting respectively.

2) Co-located energy harvester and information receiver. This requires only one set of antenna for both energy and data reception. It can be implemented using two architectures: power splitting and time splitting. In power splitting part of the received power is utilized for energy harvesting purposes and the other for simultaneous data detection. In time splitting, the complete received RF signal is utilized alternately for data detection (energy consumption) for a fixed time duration and for energy harvesting for another fixed time duration.

The unified model of the RF energy harvesting process of these architectures is given in Eq. (1) [30]

$\mathrm{E}_{\mathrm{h}}=\alpha \eta|\mathrm{h}|^{2} \mathrm{E}$

where $h$ is channel condition between the RF energy harvester and the RF energy source

$|\mathrm{h}|^{2} \mathrm{E}$ represents the energy of the received RF signals.

$0<\alpha \leq 1$ is the time switching ratio or power splitting ratio that is used for energy harvesting.

Existing literature assumes that energy harvested in current time slot can only be utilized in the subsequent time slots because of the half duplex constraint [33]. Because of this before performing any cognitive functions the CR to checks the residual energy levels in the above architectures.

Additional cognitive functionality in the form of harvesting strategies are requered, for instance, in the time switching architectures to specify whether to harvest and how long to harvest. For example if the battery used for energy storage in the energy harvester is charged to its capacity, the CR will benefit by not assigning any more time slots to energy harvesting.

Also it seems that no single power source is sufficient for the energy requirements of a CR. For energy harvesters to be a viable alternative, a combination of sources need to be harvested in the same device depending on its applications.

\section{Conclusion}

Harvesting energy from ambient energy sources is not only advantageous in terms of sustainable development but also in terms of less maintenance requirements (changing batteries etc) and zero recurring costs. Various ambient energy sources are described and its seen that the output power of all of them as a small sized(physically) energy harvester is very less. So either we need to work on increasing their efficiencies or on devising methods for hybrid energy harvesters which can utilize multiple energy sources to generate required higher levels of power. In CR systems RF energy harvesting can not only be performed using unintended signals(signals not containing data for the CR) for example GSM, WiFi(if applicable) which are omnipresent in urban environments but also on intended signals (signals containing data for CR). Two models are outlined for RF energy harvesting in $\mathrm{CR}$.

\section{References}

[1] Atelier Dna, design laboratory, atelierdna.com/windstalk, 2010

[2] W. H. Bloss, F. Pfisterer, "Advances in Solar Energy Technology: Proceedings of the Biennial Congress of the International Solar Energy Society, Hamburg, Federal Republic of Germany, 13-18 September 1987', p. 1896

[3] Danick Briand, Eric Yeatman, Shad Roundy, "Micro Energy Harvesting” John Wiley \& Sons, p. 277

[4] FaridUllah Khan and Izhar, "State of the art in acoustic energy harvesting", Journal of Micromechanics and Microengineering, Volume 25, Number 2, January 2015

[5] Texas Instruments, Energy Harvesting - White paper 2009

[6] Farid Ullah Khan and Izhar," State of the art in acoustic energy harvesting", Journal of Micromechanics and Microengineering, Volume 25, Number 2, 2015

[7] John G. De Steese Donald J. Hammerstrom Lawrence A. Schienbein," Electric Power from Ambient Energy Sources", technical report prepared for the U.S. Department of Energy under Contract DE-AC0676RL01830

[8] A. Kurs et al., ,„Wireless power transfer via strongly coupled magnetic resonances, ${ }^{\text {eee }}$ Science, vol. 317, no. 5834, pp. 83-86, Jun. 2007.

[9] H. Shoki, „Issues and initiatives for practical deployment of wireless power transfer technologies in Japan, ${ }^{\text {eee }}$ Proc. IEEE, vol. 101, no. 6, pp. 1312-1320, Jun. 2013.

[10] M. Pinuela, D. C. Yates, S. Lucyszyn, and P. D. Mitcheson, ,Maximising DC to load efficiency for inductive power transfer, ${ }^{\text {eee }}$ IEEE Trans. Power Electron., vol. 28, no. 5, pp. 2437-2447, May 2013.

[11] J. Lawson, M. Pinuela, D. C. Yates, S. Lucyszyn, and P. D. Mitcheson, „, Long range inductive power transfer

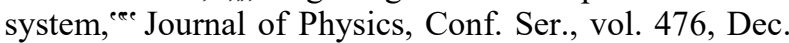
2013, 012005.

[12] A.B. Kurs, A. Karalis, R. Moffatt, J.D. Joannopoulos, P.H. Fisher, and M. Soljacic, "Wireless Power Transfer via Strongly Coupled Magnetic Resonances", Science, 317, pp. 83-86, (2007).

[13]A. Karalis, J.D. Joannopoulos, and M. Soljacic, "Efficient Wireless Non-radiative Midrange Energy Transfer", Ann. Phys., 323, pp. 34-48, (2008); published online April 2007.

[14] J.D. Joannopoulos, A. Karalis, and M. Soljacic, "Wireless Non-Radiative Energy Transfer", U.S. Patent 


\section{International Journal of Science and Research (IJSR) \\ ISSN (Online): 2319-7064}

Index Copernicus Value (2013): 6.14 | Impact Factor (2015): 6.391

Numbers $7,741,734 ; 8,022,576 ; 8,084,889 ; \quad$ and $8,076,800$.

[15] A. Karalis, A.B. Kurs, R. Moffatt, J.D. Joannopoulos, P.H. Fisher, and M. Soljacic, "Wireless Energy Transfer", U.S. Patent Numbers 7,825,543 and 8,097,093.

[16] J. S. Seybold, Introduction to RF Propagation. New York, NY, USA: Wiley, 2005, pp. 134-162.

[17] Allen M. Hawkes, Alexander R. Katko and Steven A. Cummer, "Microwave metamaterial with integrated power harvesting functionality", Applied Physics Letters vol 103, 163901 (2013)

[18] Guoqing Fu and Sameer Sonkusale, "Broadband Energy Harvesting Using a Metamaterial Resonator Embedded With Non-Foster Impedance Circuitry", arXiv preprint arXiv:1411.0662, 2014

[19] K. Finkenzeller, RFID Handbook, 2nd ed. New York, NY, USA: Wiley, 2003, pp. 271-271.

[20]R. J. Vyas, B. S. Cook, Y. Kawahara, and M. M. Tentzeris, ,E-WEHP: A batteryless embedded sensorplatform wirelessly powered from ambient digital-TV signals, ${ }^{\text {eee }}$ IEEE Trans. Microw. Theory Tech., vol. 61, no. 6, pp. 2491-2505, Jun. 2013.

[21]R. Shigeta et al., ,Ambient RF energy harvesting sensor device with capacitor-leakage-aware duty cycle control, ${ }^{\text {ere }}$ IEEE Sensors J., vol. 13, no. 8, pp. 29732983, Aug. 2013.

[22]A. Dolgov, R. Zane, and Z. Popovic, „Power management system for online low power RF energy

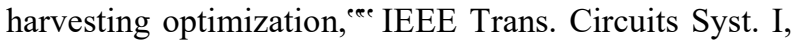
Reg. Papers, vol. 57, no. 7, pp. 1802-1811, Jul. 2010.

[23] D. Masotti, A. Costanzo, M. D. Preta, and V. Rizzoli, ${ }_{,}$, Genetic-based design of a teta-band high-efficiency radio-frequency energy harvesting system, ${ }^{\text {eec }}$ IET Microw. Antennas Propag., vol. 7, no. 15, pp. 12541263, Dec. 2013.

[24]M. Pinuela, P. D. Mitcheson, and S. Lucyszyn, ,Ambient RF energy harvesting in urban and semiurban environment, eee IEEE Trans. Microw. Theory Tech., vol. 61, no. 7, pp. 2715-2726, Jul. 2013.

[25] T. Ajmal, V. Dyo, B. Allen, D. Jazani, and I. Ivanov, ,Design and optimisation of compact RF energy harvesting device for mart applications, ${ }^{\text {cee }}$ Electron. Lett., vol. 50, no. 2, pp. 111-113, Jan. 2014.

[26]H. Reinisch et al., ,An electro-magnetic energy harvesting system with $190 \mathrm{nW}$ idle mode power consumption for a BAW based wireless sensor node, ${ }^{\text {ece }}$ IEEE J. Solid-State Circuits, vol. 46, no. 7, pp. 17281741, Jul. 2011.

[27]J. Yin et al., „A system-on-chip EPC gen-2 passive UHF RFID tag with embedded temperature sensor, ${ }^{\text {eee }}$ IEEE J. Solid-State Circuits, vol. 45, no. 11, pp. 24042420, Nov. 2010.

[28]D. Yeager et al., , ,A 9A, addressable Gen2 sensor tag for biosignal acquisition, "ee IEEE J. Solid-State Circuits, vol. 45, no. 10, pp. 2198-2209, Oct. 2010.

[29] U. Karthaus and M. Fischer, , Fully integrated passive UHF RFID transponder IC with 16.7-W minimum RF input power, ${ }^{\text {ece }}$ IEEE J. Solid-State Circuits, vol. 32, no. 10, pp. 1602-1608, Oct. 2003.

[30] Xueqing Huang; Tao Han; Ansari, N., On GreenEnergy- Powered Cognitive Radio Networks,
Communications Surveys \& Tutorials, IEEE, Year: 2015, Volume: 17, Issue: 2

[31]R. Zhang and C. K. Ho, "MIMO broadcasting for simultaneous wireless information and power transfer", IEEE Trans. Wireles Communication, vol 12, no. 5, May 2013

[32] S. Luo, R. Zhang and T. J. Lim, "Optimal save then transmit protocol for energy harvesting wireless transmitters", IEEE Transaction Wireless Communication, vol. 12, no. 3, March 2013 